\title{
Single-Trial Detection of Event-Related Potentials with Integral Shape Averaging: An Application to the Elusive N400
}

\author{
G. Rocco*, H. Rix, J. Lebrun, S. Guetat, L. Chanquoy, O. Meste, and M.-N. Magnié-Mauro
}

\begin{abstract}
The estimation of Event-Related Potentials (ERPs) from the ambient EEG is a difficult task, usually achieved through the synchronous averaging of an extensive series of trials. However, this technique has some caveats: the ERPs have to be strictly time-locked with similar shape, i.e. emitted with the same latency and the same profile, with minor fluctuations of their amplitudes. Also, the method requires a huge number of valid trials $(\sim 100)$ to efficiently raise the ERPs from the EEG trials. In the case of cognitive ERPs, as with the N400, the delivered stimulus has to be different for each trial, the latencies are varying, and the number of available trials is usually low. In this paper, an alternative method, coined Integral Shape Averaging (ISA) and its derivatives are detailed. ISA is robust to varying latencies and affine transforms of shape. Furthermore, a new method coined ISAD can be derived to extract ERPs even from a single trial experiment. The aim here is to illustrate the potential of ISAD for N400 component extraction on real EEG data, with emphasis on its general applicability for ERPs computation and its major assets like reduced experimental protocol. Some insights are also given on its potential use to study ERP variability, through shape and latency.
\end{abstract}

Clinical Relevance - The proposed algorithm aims to be a helpful tool in clinical practice to analyze and interpret evoked responses in real experimental settings, especially for particularities in neurology.

\section{INTRODUCTION}

Event-related potentials (ERPs) are classically estimated through the synchronous averaging of a series of recordings (EEG) obtained by the repetition of stimuli of the same nature, e.g. auditory or visual $[1,2]$. Ideally, the ERP latency and shape is assumed constant among all the trials, thus the averaging of an increasing number of trials will enhance the event-related response and decrease the randomness (non-event-related) of the EEG activity. However, there are some cases in which synchronous averaging may lead to a biased estimation of the ERPs components. For instance, when extracting the highly elusive N400 component, a negative-going deflection around $400 \mathrm{~ms}$ which is typically evoked in response to semantic incongruity [3], the synchronous averaging method usually returns a shallow signal that may not be a genuine ERP. One of the main reasons lies in the fact that the stimulus, e.g. a word, must be changed at each trial [4]. Consequently, each computed ERP may be altered in its latency, its width associated with a scaling factor on the abscissas or more generally in its shape. An alternative to synchronous averaging when the signals are made positive is Integral Shape Averaging (ISA) [5,6]. If all the signals have the same shape

* Research was supported by ANR-15-IDEX-01 under Project HP, IDEX UCA-JEDI and H2020 MSCA COFUND programme "BoostUrCareer" under grant agreement 847581 .

* Corresponding author is G. Rocco - Email: grocco@i3s.unice.fr despite jitter and width variations, the ISA technique returns an averaged signal with the same common shape. Thus, unlike synchronous averaging, ISA overcomes the jitter-related issues that cause peak smoothening. Also, by using the ISA approach with some resampling techniques, it is now possible to estimate the shape variability among trials, by extracting ERPs from a single trial. This method, named ISAD was already tested in [7] with simulations for Gaussians estimation drowned in white noise and yielded promising results [7]. Here we validate this approach to efficiently extract elusive ERPs components, like the N400, from ambient real-world EEG signals. In the following, the experimental conditions for data acquisition are described, and a short recall of ISA and ISAD is given, followed by a description of the results achieved and the comparison with the gold standard method, i.e. synchronous averaging.

\section{MATERIAL \& METHODS}

\section{A. Data acquisition}

Twenty continuous EEGs of 2 hours each were recorded from a cohort of young subjects at the University Hospital of Nice (CHU-Pasteur) using an ANT Neuro Eego/Mylab device with 32 electrodes $\mathrm{Ag} / \mathrm{AgCl}$ mounted in an elastic Waveguard cap with 10/20 standard positions. From this set, we randomly selected one case study aged 10 to apply our new methods. Electrooculogram (EOG) was monitored using bipolar electrodes laterally to the left and right eyes (horizontal EOG) and above and below the left eye (vertical EOG). All electrodes (except for the bipolar channels) were single referenced and electrode impedance never exceeded $5 \mathrm{k} \Omega$. EEG was sampled at $2048 \mathrm{~Hz}$. The clinical trial was performed in compliance with laws and institutional guidelines and was approved by the UCA's CERNI (Comité d'Ethique pour les Recherches Non Interventionnelles).

\section{B. Experimental design}

The data were collected using a classical semantic priming task to elicit the N400 component. The whole experimental

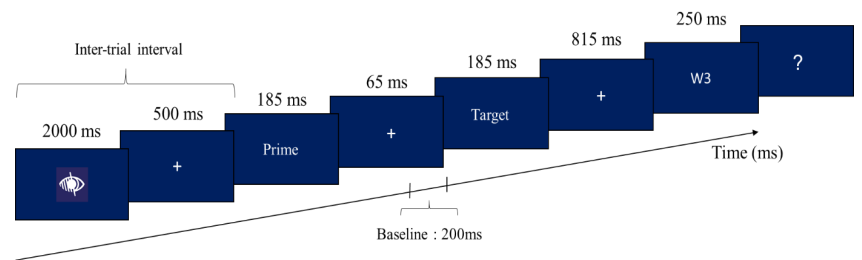

Figure 1. Outline of the experimental protocol

All authors are with Université Côte d'Azur, Nice, France. G.R, H.R, J.L, O.M are also with lab. I3S (CNRS/UCA); S.G., L.C., M.N.M.M. are with lab. BCL (CNRS/UCA); M.N.M.M is also with CHU Nice. 
protocol is illustrated in Fig. 1. Each trial consisted in the central presentation of a first word (the prime), followed by the second word (the target) with a stimulus-onset asynchrony of $250 \mathrm{~ms}$. Then, a third word is presented ("W3" in Fig. 1), which could possibly be the repetition of one of the first two. The N400 component was recorded from the onset of the target word. This experimental protocol is well-known for its ability to elicit strong N400 responses due to a semantic priming phenomenon [8]. An accelerated processing of the target word can be observed when it is preceded by an associatively related prime word, compared to when it is preceded by an unrelated prime word. The semantic relatedness of each pair was manipulated such that in half of the trials, the prime and target words had a strong associative links (e.g., « dog - kennel »), while in the other half, they had no links (semantic, orthographic or phonological, e.g., « fork - eraser $»)$. To constitute the pairs of words with the strong associative link, 87 pairs of french words were pre-selected from the standards of verbal associations for children [9]. Afterwards, 24 children (mean age $=10$ years; S.D. $=10$ months; range $=8$ years and 7 months -11 years and 5 months) were asked to rate, on a 7-point Lickert scale, the degree of similarity in terms of physical and functional characteristics $(7=$ very similar, $1=$ not at all similar). Sixty pairs were retained among those judged the least similar with an average degree of overlapping characteristics as 1.81 (S.D. $=0.44$; range $=1.15-2.95$ ). All the targets were matched on length and written word-frequency in common texts [10]. To avoid fatigue and memory biases, one hundred similar variations of this trial were recorded.

\section{Data analysis}

Fifty epochs ([-200 ms, $800 \mathrm{~ms}]$ with the target stimulus onset at $0 \mathrm{~ms}$ ) were selected from the signal recorded at the $\mathrm{CPz}$ electrode, assessed to have the highest $\mathrm{N} 400$ response [11], with referencing to the mastoids. Epochs with eye blinks, amplifier clipping, and muscle artifacts were excluded from the analyses by visual inspection. The signals were band-pass filtered at $0.1-30 \mathrm{~Hz}$ with half amplitude cut-off.

\section{THEORY}

Before describing our analysis, let us recall the theoretical concept behind ISA and ISAD.

\section{A. Integral Shape Averaging}

Considering a series $\left\{f_{i}(t)\right\}$ of positive signals which are assumed to have the same shape, i.e. with all envelopes linked by affine transformations to a common prototype, their integral functions normalized by the areas under the curves may be seen as cumulative distribution functions (cDF). Namely, let $S=\left\{f_{i}(t) \mid i=1, \ldots, \mathrm{N}\right\}$ be a set of real positive signals, $F_{i}^{\star}(T)$ the cumulative distribution function of signal $f_{i}(t)$ is defined as:

$$
\begin{gathered}
\int_{-\infty}^{T} f_{i}(t) d t=: F_{i}(T) \\
F_{i}^{\star}(T):=F_{i}(T) / F_{i}(+\infty)
\end{gathered}
$$

As cDF, it is monotonically increasing and spans values from 0 to 1 . Let us now average the inverse cDFs of all signals:

$$
\forall y \in[0,1], \quad t_{\mu}(y):=\frac{1}{N} \sum_{i=1}^{N}\left(F_{i}^{\star}\right)^{-1}(y)
$$

By plotting all the $y$ values as function of the computed $t_{\mu}$, we get an "integral average" $\operatorname{cDF} F_{\mu(S)}^{*}(t)$ of all signals. Then, the ISA signal is simply the "mean" density function (pDF) obtained by differentiating the "mean" $\mathrm{cDF} F_{\mu(S)}^{*}(t)$ in time $t$. The reader is referred to $[6,7]$ for a more thorough description of the method. The main property of the ISA signal is that it has the same common shape as the initial signals $\left\{f_{i}(t)\right\}$, with mean positioning in time and mean width for shape. It is important to stress out that this property is usually not obtained with synchronous averaging that requires strict timelocked signals, higher number of trials and still produces typical artifacts like peaks smoothing [5].

\section{B. ISA of delayed windows of a single trial (ISAD)}

The main limitations of ISA are that artifacts may appear when the shape of the signals varies too much from trial to trial, or when the number of trials is too small even for ISA (typically less than 20, in contrast with extraction of ERPs by synchronous averaging that requires at least 100 trials). A newly derived method, coined ISAD, was designed to apply ISA on an artificially recreated larger series of same-shape signals from a single trial using a resampling technique $[12,13]$. Precisely, let $W_{0}$ be our window of interest in the available trial, inside which most of the signal lies in time (in our case 500 samples), and two other shorter windows $W_{1}$ and $W_{2}$ of same length (200 samples) filled with randomly generated white noise (or potentially some EEG baseline noise) contiguously added before and after $W_{0}$. By sliding the sequence of windows $\left[W_{1}, W_{0}, W_{2}\right]$ on the original single trial (see Fig. 2), with each new displacement of the windows containing a slightly different part of the signal of interest bordered with new sequences of noise, one gets a new series of $\sim 250$ epochs or signals on which ISA can be applied. In case some more genuine trials are available, another ISA (or other analysis tools) may be applied again on the whole series of ISAD signals obtained from each trial. As N400 ERPs are difficult to evoke (fatigue and memory issues) and synchronous averaging requires $\sim 100$ trials to get a decent ERP, this ISAD single trial approach provides an interesting new tool to study ERPs, even more when ISA of the ISADs is applied on a small number of genuine trials.

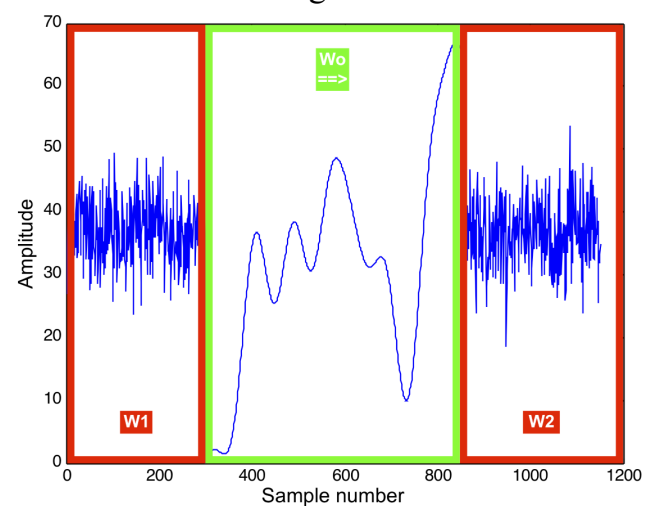

Figure 2. A statistical resampling method: creating a larger set of signals for ISA processing by sliding $\sim 250 \mathrm{x}$ a triplet of windows on a single trial 


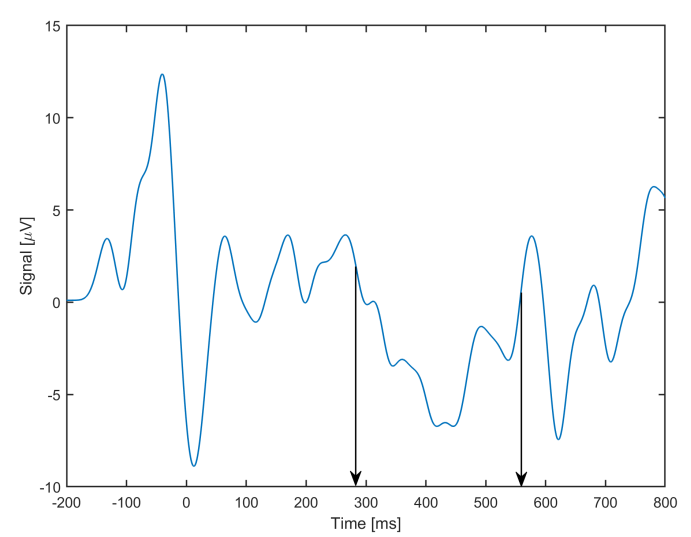

Figure 3. Synchronous average of the 50 trials

\section{Application to a real cognitive ERP: N400 component}

Fig. 3 shows the synchronous averaging of the 50 trials. The N400 component is the negative wave with a minimum around $400 \mathrm{~ms}$, with amplitude around $-8 \mu \mathrm{V}$ (compared with the $100 \mu \mathrm{V}$ amplitude for the original EEG signal). From this curve we extract an interval $\left[T_{1}, T_{2}\right]$ in which individual N400 ERPs are most likely to be localized. Here, we took $T_{1}=285$ $\mathrm{ms}$ and $T_{2}=555 \mathrm{~ms}$ [14].

A central assumption underlying ISA and ISAD is that the considered signals are positive, which is intrinsically not the case of EEG. Several transformations can be used to obtain positive signals, e.g. the analytic amplitude from the Hilbert transform of the EEG or more simply by offset addition in amplitude. Indeed, it can be shown that shape equality in a set of signals is not altered if an offset equal to the minimum of the signal is added. Thus, each trial can be made positive by an offset addition with additional detrending to avoid baseline drifts. In order to study some within-subject variability in the ERPs, one may compare the shapes of the N400 components obtained by ISAD on trials $k$ and $l$ (with $S_{k}$ and $S_{l}$ being the set of artificial signals obtained with resampling) using the shape similarity criterion based on the Distribution Function Method (DFM) $[15,16,17]$, i.e. a "linear MMSE"-like error measuring how close the two $\operatorname{cDFs} F_{\mu\left(S_{k}\right)}^{*}(t)$ and $F_{\mu\left(S_{l}\right)}^{*}(t)$ may be transformed one into another using an affine mapping, as this would gives the same shape for the underlying signals

$\Delta_{k . l}:=\min _{\alpha>0, \beta}\left\|F_{\mu\left(s_{l}\right)}^{* 1} \circ F_{\mu\left(s_{k}\right)}^{*}(t)-(\alpha t+\beta)\right\|^{2}$

with $\|$.$\| being \mathrm{L}^{2}$ norm on the selected interval $\left[T_{1}, T_{2}\right]$ where the N400 components lie. The latency of a single trial is estimated by its mean time according to the following expression:

$$
t_{\mu}:=\int_{T_{1}}^{T_{2}} t \cdot y(t) d t / \int_{T_{1}}^{T_{2}} y(t) d t
$$

where $y(t)$ may be the synchronous averaging of all trials or any ISAD of a single trial.

\section{Results}

Fig. 4 shows some examples of five N400 components extracted separately from 5 different single trials. As expected for cognitive processes, a certain degree of variability of the

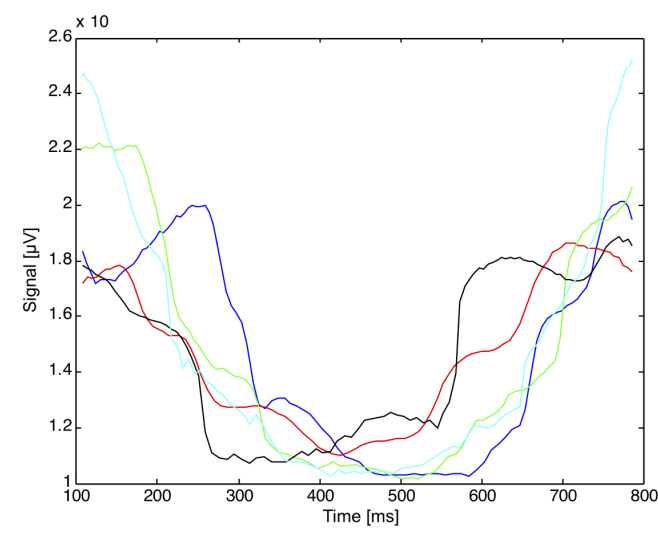

Figure 4. An example of variability between five ISAD signals

signal shapes and amplitudes is obvious. Now, using the Distribution Function Method (DFM), we computed the shape similarity differences between the obtained ISAD signals and grouped them inside the symmetric matrix $\Delta$. Fig. 5 shows the shape distances of every ISAD signal from a particular one: a higher variability in shape may be noticed between trials 35 and 40, likely attributed to some psychophysiological annotated event. In fact, this estimator could be useful to analyze the patient's status based on abrupt changes of the ISAD shape. A latency of $428.4 \mathrm{~ms}$ was computed according to (5) for the N400 component in the synchronous average (Fig.3) while the latencies of the ISAD components plotted in Fig. 6 display again a high variability. The mean value of the 50 latencies is $423.5 \mathrm{~ms}$ with a standard deviation of $40 \mathrm{~ms}$ and a range of $80 \mathrm{~ms}$. As suspected, the withinsubject variability of the latencies is again not negligible, demonstrating once more how the application of synchronous averaging in real settings may be sub-optimal with some loss of information, typical peaks smearing and even artifacts in the curves obtained. To further study the shape variability of the ISAD signals along time on the 50 trials, we proposed to use an ISA of moving sets of 5 consecutive ISAD signals to estimate the level of changes in shape similarity along time in the experiment. Fig. 7 displays four consecutive ISA of sets of ISAD which are highly different. This method allows to track discontinuities easily and precisely in shape and the evolution of latencies along time, that may be correlated to the patient psycho-physiological status. Now, it would be interesting to validate this information with objective measurements and annotations of the patient's status and to

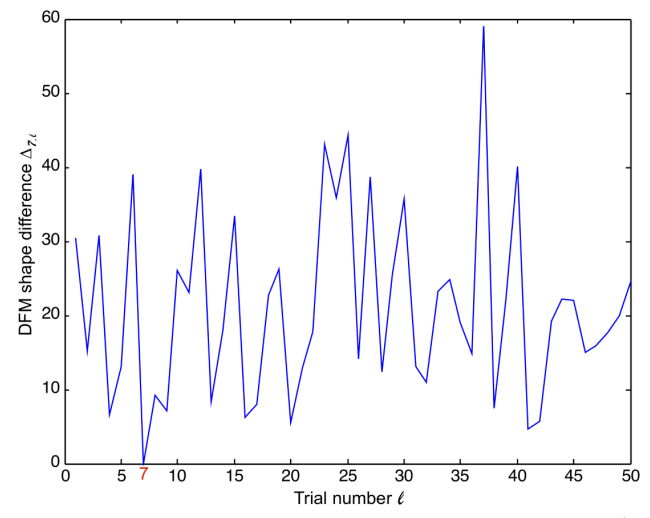

Figure 5. Shape differences $\Delta 7, l$ between all the trials and the $7^{\text {th }}$ one: on the $\mathrm{x}$ axis, the number of trial; on the $\mathrm{y}$ axis the value of $\Delta_{7, l}$ 


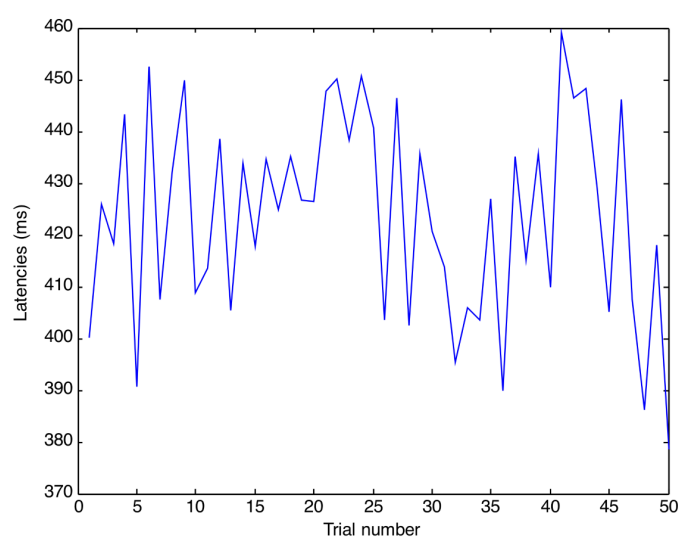

Figure 6. Latency estimations of the N400 component for 50 ISAD signals

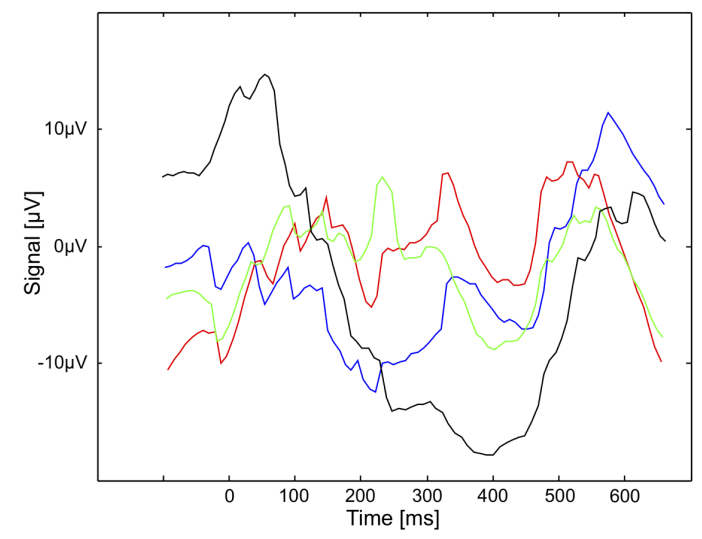

Figure 7. ISA of moving sets of 5 consecutive ISAD signals

compare it with other new alternative methods based on Machine Learning [18] that also promise single-trial analysis of latencies.

\section{DISCUSSION AND CONCLUSION}

In this paper, we introduced several ISA-based methods as alternatives to synchronous averaging for the extraction of cognitive ERPs and other features like shape similarity and latency from single trials. In particular, the advantage of our methods is twofold: on one side, it allows to consistently reduce the number of trials needed to get accurate ERPs estimations, thus drastically reducing the duration of the experimental protocol and alleviating the risk of any learning/memory or fatigue phenomenon; on the other side, it preserves some physiologically relevant information related to the patient's status, which are lost with synchronous averaging. In fact, both the estimated latencies for each ISAD trial and the moving average of consecutive trials confirms a greater than expected within-subject variability during the same experimental session and sprouts a whole new field of study so as to understand the underlying subjective psychophysiological reasons that may be related to changes in stimuli, vigilance, attention, or task strategy [19]. Thus, clinicians could retrieve important information for their diagnosis, especially to achieve a finer assessment of particularities in neurology.

In conclusion, this work presents a novel way to extract and analyze cognitive ERPs from single trials, taking into account the variabilities in shapes and latencies. The proposed algorithm could be quite helpful in clinical investigations to gain insights about the behavioral aspects in brain studies and also to optimise experimental protocols.

\section{REFERENCES}

[1] J. Polich and M. D. Comerchero, "P3a from visual stimuli: typicality, task, and topography," Brain Topogr., vol. 15, no. 3, pp. 141-152, Mar 2003.

[2] N. K. Squires, K. C. Squires, and S. A. Hillyard, "Two varieties of longlatency positive waves evoked by unpredictable auditory stimuli in man," Electroen. Clin. Neuro., vol. 38, no. 4, pp. 387-401, Apr 1975.

[3] M. Kutas, and S. A. Hillyard, "Reading senseless sentences: Brain potentials reflect semantic incongruity," Science, vol. 207, no. 4427, pp. 203-205, Jan 1980.

[4] S. J. Luck, "An introduction to event-related potentials and their neural origins," in An introduction to event-related potential technique, MIT Press, Cambridge, 2005, ch. 1, pp. 1-14.

[5] H. Rix, O. Meste, and W. Muhammad, "Averaging signals with random time shift and time scale fluctuations," Method. Inform. Med., vol. 43 , no. 1, pp.13-16, 2004

[6] S. Boudaoud, H. Rix, and O. Meste, "Integral shape averaging and structural average estimation: a comparative study," IEEE Trans. Sig. Proc., vol. 53, no. 10, pp. 3644-3650, Sep 2005.

[7] H. Rix, "Integral Shape Averaging applied to delayed windows of the same signal," Internal Research Report, Laboratoire I3S UCA, 2018. 〈hal-01831897〉

[8] J. H. Neely, "Semantic priming effects in visual word recognition: A selective review of current findings and theories," in Basic processes in reading: Visual word recognition, Lawrence Erlbaum, Hillsdale, NJ, 1991, ch. 9, pp. 264-336.

[9] F. De La Haye, "Normes d'associations verbales chez des enfants de 9, 10 et 11 ans et des adultes," L'année psychologique, vol. 103, no. 1, pp. 109-130, 2003.

[10] R. Peereman, B. Lété, and L. Sprenger-Charolles, "Manulex-infra: Distributional characteristics of grapheme-phoneme mappings, infralexical and lexical units in child-directed written material," Behav. Res. Methods, vol. 39, pp. 593-603, Aug 2003.

[11] L. Amoruso, et al., "N400 ERPs for actions: building meaning in context," Front. Hum. Neurosci., vol. 7, no. 57, Mar 2013.

[12] N. A. Parks, et al., "Bootstrap signal-to-noise confidence intervals: an objective method for subject exclusion and quality control in ERP studies," Front. Hum. Neurosci., vol. 10, no. 50, Feb 2016.

[13] K. Kotowski, K., Stapor, and J. Leski, "Improved robust weighted averaging for event-related potentials in EEG," Biocybern. Biomed. Eng., vol. 39, no. 4, pp. 1036-1046, Oct 2019.

[14] D. Deacon, et al., "Variation in the latencies and amplitudes of N400 and NA as a function of semantic priming," Psychophysiology, vol. 32, no. 6, pp, 560-570, Nov 1995.

[15] H. Rix and J.-P. Malengé, "Detecting small variations in Shape," IEEE Trans. Syst., Man and Cyber., vol. 10, no. 2, pp. 90-96, Feb 1980.

[16] H. Rix and J.-P. Malengé, "Detection of an impurity (1\%) at low resolution (0.25)," J. High Res. Chromatog., vol. 3, no. 4, pp. 172-176, Apr 1980.

[17] N. Żołek, H. Rix, and M. Botwicz, "Analysis of estimation of optical properties of sub superficial structures in multi layered tissue model using distribution function method," Comput. Meth. Prog. Bio., vol. 183, article 105084, Jan 2020.

[18] I. Hardiansyah, V. Pergher, and M. M. Van Hulle, "Single-trial EEG responses classified using latency feature," Int. J. Neural Syst., vol. 30, no. 6, article 2050033, Jun 2020.

[19] V. Legrain, et al., "Attentional modulation of the nociceptive processing into the human brain: selective spatial attention, probability of stimulus occurrence, and target detection effects on laser evoked potentials," Pain, vol. 99, no. 1-2, pp. 21-39, Sep 2002. 